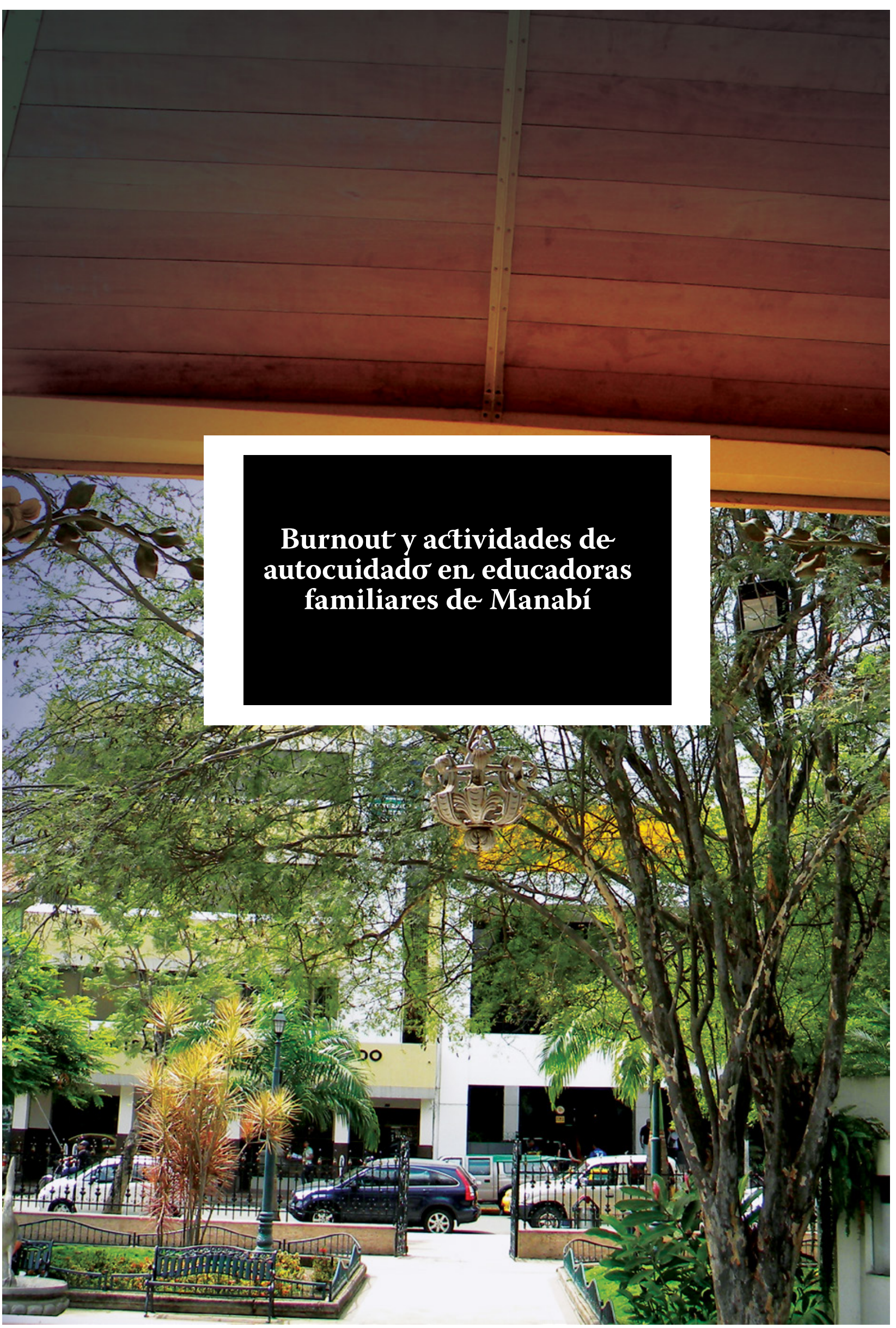




\title{
BURNOUT Y ACTIVIDADES DE AUTOCUIDADO EN EDUCADORAS FAMILIARES DE
} MANABí

\section{BURNOUT AND SELF-CARE ACTIVITIES IN FAMILY EDUCATORS OF MANABí}

\section{RESUMEN}

Para contribuir a la comprensión del "burnout" y el autocuidado de las educadoras familiares de Manabí, se caracterizó socioeconómicamente la población, se estimó la presencia del síndrome y se determinó la aplicación de actividades de autocuidado. La metodología consideró un enfoque cuanti-cualitativo, con alcance descriptivo, un diseño no-experimental transversal, se aplicó como técnicas e instrumentos: ficha sociodemográfica, cuestionario para actividades de autocuidado, y el Inventario de "Burnout" de Maslach. La población estuvo compuesta por una muestra de doscientas treinta y seis educadoras familiares en formación dual. Se encontró que la mayoría de ellas presentan el síndrome: de forma leve, y en menor porcentaje moderada, y severa presencia. Se halló que se somatiza más en nuca-espalda y cabeza, y que las actividades de autocuidado más practicadas son la actividad física, "decir no cuando es necesario", y dormir; además, la mayoría está de acuerdo en practicar actividades de autocuidado y se considera capaz de abandonar un mal hábito. Se concluye que existe un porcentaje considerable de la población que está en riesgo pues tiene presencia leve del síndrome, pero no tiene conciencia de padecerlo, mientras que para las demás participantes sería necesaria la intervención específica con estrategias de autocuidado y tratamiento profesional.

PALABRAS CLAVE: Autocuidado, burnout, educadoras familiares, formación dual.

Copyright @ Revista San Gregorio 2019. ISSN: 1390-7247; eISSN: 2528-7907 @

\begin{abstract}
To contribute to the understanding of the "Burnout" and self-care of family educators of Manabí, was characterized socioeconomically the population, it was felt the presence of the syndrome and determined the implementation of self-care activities. The methodology considered a quanti-qualitative approach, with descriptive, a cross-sectional non-experimental design, applying techniques and instruments: a socio-economic tab, a questionnaire for self-care activities, and the Maslach's Burnout Inventory. The population was composed of a sample of two hundred and thirty-six family educators on dual training. It was found that most of them present the syndrome: mild form, and lower percentage of moderate and severe presence. It was found that the place of the body with more somatization is neck-back and head, and that the most widely practiced self-care activities are physical activity, "say no when necessary," and sleep; in addition, the majority was agree to practice self-care activities and consider themselves capable of abandoning an unhealthy habit. It is concluded that there is a considerable percentage of the population that is at risk because it has mild presence of the syndrome, but not aware of suffering, while for the other participants it would be necessary the intervention specified with self-care and professional treatment strategies.
\end{abstract}

KEYWORDS: Burnout, self-care, family educators, dual training.

Copyright @ Revista San Gregorio 2019. ISSN 1390-7247; eISSN: 2528-7907 @

EnriQue Quiroz Zambrano

ه

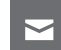

Ministerio de Educación. Manta, Manabí. Ecuador

psicoenrique08@hotmail.com

\section{Eva Ganchozo Macías}

全

M evamgm-2@hotmail.com

Boris Mariano Mero Casanova

소

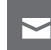

Freelance. Ingeniero en Sistemas. Portoviejo. Manabí. Ecuador.

borismero@gmail.com

ARTÍCULO RECIBIDO: 9 DE JUNIO DE 2018 


\section{INTRODUCCIÓN}

La incidencia del Burnout a nivel global es innegable, "el cual se ha convertido en un fenómeno de interés general en la sociedad moderna" (Díaz \& Gómez, 2016, p. 114). Numerosas investigaciones, centradas en las manifestaciones clínicas del estrés, es decir, en el estrés negativo o distrés, han encontrado que afecta la calidad de vida y las distintas áreas de funcionamiento social, familiar, académica, laboral (Quiceno \& Vinaccia, 2007). Uno de los factores más preponderantes en el desarrollo del estrés es el "síndrome del quemado" o Burnout (en inglés).

El Burnout, descrito primero por Freudenberger (1974) fue definido operacionalmente luego por Maslach y Jackson (1981) como un síndrome psicológico de agotamiento emocional, despersonalización y reducción en la realización personal en el trabajo y con las personas, que hace, que quienes lo padecen se sientan descontentas consigo mismos e insatisfechos con sus logros en el trabajo; todo esto resulta según estos autores potencialmente muy grave para el personal, los clientes y las instituciones en las que interactúan.

Uno de los expertos en el tema, Pedro Gil-Monte, fue el primero en traducir al español el término (Burnout) como "Síndrome de Quemarse por el Trabajo (SQT)", citado por (Díaz B. \& Gómez, 2016) que lo define como:

(...) una respuesta psicológica al estrés laboral crónico, de carácter interpersonal y emocional, que aparece en los profesionales de las organizaciones de servicio que trabajan en contacto con los clientes o usuarios de la organización, y se caracteriza por un deterioro cognitivo que consiste en una pérdida de la ilusión del trabajo, el desencanto profesional o la baja realización personal en el trabajo, por un deterioro afectivo caracterizado por agotamiento emocional y físico y por actitu- des y conductas negativas hacia los clientes y hacia la organización. (p.116).

El síndrome de Burnout fue declarado en el año 2000 por la Organización Mundial de la Salud (OMS) como un factor de riesgo laboral por su capacidad para afectar la calidad de vida, salud mental e incluso hasta poner en riesgo la vida (Saborío \& Hidalgo, 2015). "La OMS al hacerse eco de la situación declaró al síndrome de Burnout como uno de los cinco problemas sanitarios más graves de la actualidad" (Díaz, Radler, Amadeo \& Guerrero, 2011, p.3).

$\mathrm{Si}$ bien en Ecuador existe un considerable número de estudios sobre el burnout: (Quevedo, 2012); (García, Delgado \& Lazo, 2018); (Brito, Cabrera \& Cedilllo, 2012); (Cárdenas, 2013); (Cruz, Guanga \& León, 2014); (Pérez, 2017); (Caicedo, 2018); (Ramírez, 2015); (Vidal, Nicasio \& Pacheco, 2010); (Garzón, 2017) (Ramírez, 2017 a); (Ramírez, 2017 b); (Vivanco, Sánchez, Maldonado \& Erique, 2018); (Vilcahuano Germán, 2015); (Gómez, Algora, Suasnavas, Silva \& Vilaret, 2016); (Costales, 2018); (Barragán, 2016); (Menéndez, Calle, Delgado, Espinel \& Cedeño, 2018); (Pesántez, 2015); entre otros, la mayoría de estos corresponden a estudios sobre profesionales o personal en formación que trabaja en salud, con solo unos pocos estudios sobre otras poblaciones; este hecho es similar en Latinoamérica, región en la que las investigaciones sobre burnout presentan en su mayoría validaciones de instrumentos o estudios en personas vinculadas al sector asistencial y de salud (Díaz \& Gómez, 2016) (Pando, Aranda \& López, 2015) y así también en otras regiones de Europa o Norteamérica (Clipa, 2017); (Zaluska, Ślazyk-Sobol \& Kwiatkowska-Ciotucha, 2018); (Yawen \& Zhen, 2018); (Cooper y otros, 2016); (Hall, Johnson, Watt, Tsipa, \& O'Connor, 2016) quedando por fuera quizá, otros grupos humanos que pueden estar expuestos al burnout.

Uno de estos grupos de profesionales y personas en formación que trabajan en contacto permanente con un cuantioso número de seres humanos son las Educadoras Familiares de Manabí, quienes desde los programas denominados: Creciendo con nuestros Hijos $(\mathrm{CNH})$, y Centros infantiles del Buen Vivir (CIBV) del Ministerio de Inclusión económica y social, MIES, atienden y apoyan el desarrollo infantil Integral de más de 49 mil niñas y niños (MIES, 2015), con un grupo humano de 
educadoras que supera ampliamente las mil personas.

De estas Educadoras, 357 se han matriculado en la carrera de Tecnología en Desarrollo Infantil Integral, ofertada por el "Instituto Tecnológico Superior Paulo Emilio Macías" (ITSPEM), a través de becas otorgadas por la Secretaría Nacional de Educación Superior, Ciencia, Tecnología e Innovación (SENESCYT) con el propósito de mejorar la calidad de los servicios de atención infantil, en el marco de la "Estrategia de Mejoramiento del Talento Humano, a través de programas de profesionalización y capacitación continua dirigidos a todo el personal responsable de la operación de las modalidades de atención infantil" (MIES, 2015, p. 2).

Dentro de estas circunstancias, estas Educadoras familiares en formación, se ven expuestas a desarrollar el síndrome de Burnout, puesto que tienen una carga de trabajo significativa en el marco de interacciones sustanciales con niñas, niños, familias, compañeros de trabajo y representantes institucionales, al mismo tiempo que se capacitan a través de la modalidad dual ${ }^{1}$, y viven la cotidianidad de sus propias familias. De allí que se impone la necesidad de identificar la presencia del Síndrome de Burnout en este grupo poblacional pues "(...) es una prioridad de la academia, sobre todo, el descubrir los diferentes malestares que viven las estudiantes, durante el proceso de formación profesional" (López-Pozos, 2014 , p. 185) y la necesidad de dar cuenta del conocimiento y aplicación de Actividades de Autocuidado ("self care", en inglés) en favor de su propia salud.

En 1982, la Organización Mundial de la Salud, OMS, definió el autocuidado como: las actividades de salud no organizadas y a las decisiones de la salud tomadas por individuos, familia, vecinos, amigos, colegas, compañeros de trabajo, etc. Así, "el autocuidado es, definitivamente, el recurso sanitario fundamental del sistema de atención de salud" (OMS, 2008) (Citado en Castillo, 2012, p. 15).

Para Prado, González, Paz, \& Romero (2014) el autocuidado:
(...) es una función reguladora del hombre que las personas deben, deliberadamente llevar a cabo, por sí solas, una actividad del individuo, aprendida por este y orientada hacia un objetivo, una conducta que aparece en situaciones concretas de la vida, y que el individuo dirige hacia sí mismo o hacia el entorno para regular los factores que afectan a su propio desarrollo y actividad en beneficio de la vida, salud o bienestar (p. 839).

Actualmente, las Actividades de Autocuidado en el lugar de trabajo se han transformado en una hoja de ruta obligatoria para las instituciones, los empresarios, trabajadores $\mathrm{y}$ profesionales y todos quienes tienen a su cargo la interacción con muchas personas, la gestión de la salud y la seguridad en el trabajo. La OMS refiere que:

El autocuidado se relaciona con todo aquello que las personas hacen por sí mismas para conservar sus condiciones de salud, cuyo propósito es el de promocionar la vida y el bienestar de los seres humanos, mientras que la gestión de la salud y la seguridad en el trabajo, está orientada a preservar la integridad de las personas, siendo conscientes de que un gran porcentaje del resultado es responsabilidad de cada uno y de su estilo de trabajo. (Citado en: Hernández, 2015, p. 80).

Así, la salud tanto física como mental, los ambientes de trabajo saludables y el bienestar común se inscriben entre los recursos más valiosos para los seres humanos, las sociedades y naciones. Pero a esta salud se le está dando una nueva perspectiva que implica ya no una posición pasiva frente a lo que nos acontece, sino la posición activa sugerida por la OMS, en la que la salud es responsabilidad de cada uno y de todos (Camacho E, 2014).

Cuando Moreno-Jiménez, González \& Garrosa (2001) decían que la novedad del concepto de burnout consistía en que no era, como tantos otros, el resultado de una exploración de laboratorio, sino la constatación de un problema real experimentado en amplios núcleos de la profesión laboral, expresaban algo que no ha perdido ni un ápice de vigencia.

1. La modalidad dual es un proceso de formación profesional, propuesto por SENESCYT, que los estudiantes realizan en dos entornos de aprendizaje: el académico y el laboral, al tiempo que, basándose en principios de autonomía, responsabilidad y autogestión, trascienden las formas tradicionales de enseñanza. 
Nuestra sociedad global se encuentra inmersa en una situación crítica, producto del declive de la economía de mercado, la vorágine tecnológica y las necesidades sociales, entre otros factores que generan el caldo de cultivo ideal para la exacerbación de los síntomas de desgaste laboral, del burnout. Frente a este escenario se presenta imprescindible y pertinente la investigación del fenómeno del "síndrome del quemado", pero ya no solo para conceptualizarlo en generalidades, sino para a través de la comprensión de la particularidad de los contextos en los que se produce, coadyuvar a establecer estrategias de afrontamiento.

En consecuencia, la presente investigación busca ampliar la comprensión del Síndrome de Burnout en las Educadoras Familiares de Manabí, constituyendo así el primer paso de un esfuerzo más amplio y profundo para contribuir a la salud mental y la calidad de vida de las/los sujetos participantes, y al buen vivir en general en relación con los entornos en los que interactúan, desde la lógica de la responsabilidad subjetiva y el autocuidado.

Desde estas perspectivas, esta investigación se torna, no solo conveniente, sino de consistente relevancia social (Hernandez Sampieri, Fernández, \& Baptista, 2010), ya que al tratarse de participantes cuya labor es la educación familiar temprana, se aporta directa e indirectamente a la comunidad y al entorno inmediato en que se implican de manera contundente, contribuyendo además al desarrollo metodológico y práctico en el abordaje de esta problemática psicosocial. Para lograrlo se partió de las siguientes preguntas de investigación: ¿Cómo se caracterizan socioeconómicamente las educadoras familiares de Manabí? ¿Presentan indicadores del síndrome de Burnout las educadoras familiares de Manabí? ¿Conocen y aplican, a sí mismas/os, actividades de autocuidado las/los sujetos participantes del estudio?

\section{METODOLOGÍA}

En cuanto a la metodología de la investigación, el presente estudio considera un enfoque mixto cuantitativo-cualitativo, un alcance descriptivo, y un diseño no experimental, transversal (Hernandez Sampieri, Fernández, \& Baptista, 2010) atendiendo a la naturaleza de los objetivos a cumplir.

\section{SUJETOS Y TAMAÑO DE LA MUESTRA}

El universo de la investigación está compuesto por 357 Educadoras Familiares inscriptas en la carrera de Desarrollo Infantil Integral $(\mathrm{N}=\mathrm{U})$. De estás, se trabajó con una muestra (n) de 236 educadoras familiares, siendo los criterios de participación: Trabajar como Educadora CNH o CIBV, estar matriculada/o bajo la modalidad de formación dual, trabajar en alguno de los cantones de Manabí, participar voluntariamente en el estudio.

\section{INSTRUMENTOS}

Se utilizó una escala previamente estandarizada: Maslach Burnout Inventory (Maslach \& Jackson, 1981) para el diagnóstico del síndrome del quemado; y se diseñó una encuesta tipo cuestionario, bajo la lógica de instrumento hecho a medida del investigador, testado a través del coeficiente de confiabilidad Alfa de Cronbach, para determinar la homogeneidad del instrumento, el cual alcanzó un índice de 0.86. El manejo de datos se realizó a través de registros consolidados en una base de datos, que permitió el análisis estadístico descriptivo mediante el uso del software estadígrafo SPSS (Statistical Package for Social Sciences - Versión 21.0).

\section{PROCEDIMIENTO}

Las etapas llevadas a efecto en este estudio fueron: revisión de la literatura existente, obtención y diseño de instrumentos, recolección de datos, procesamiento y análisis de datos, y redacción del informe de investigación. Para el trabajo con las participantes se utilizó un documento de consentimiento informado.

\section{RESULTADOS}

La presentación de los resultados se articula en tres partes, por un lado, la caracterización socioeconómica de las educadoras familiares de Manabí, por otro lado, los indicadores del síndrome de Burnout en las mismas y finalmente el conocimiento y la práctica por sí mismas/os de actividades de autocuidado en las/los sujetos participantes del presente estudio.

En cuanto a las características sociodemográficas de las personas participantes, el 98,7\% de personal encuestado en el presente estudio son mujeres y el restante hombres. La edad promedio de las personas participantes en esta investigación es de 37 años. El 38,1\% 
del total de las educadoras son casadas, mientras que un $23,7 \%$ mantienen una unión libre, el $22,2 \%$ son de estado civil solteras, $5 \%$ divorciadas, $2,9 \%$ separadas y apenas un $0,84 \%$ presentan viudez. Se determina que el $61,44 \%$ de las educadoras participantes del estudio consideraron tener una situación económica "regular", mientras que el 33,47\% considera presentar una situación económica "buena"; en tanto el $83,89 \%$ del total tienen aún a cargo el cuidado de sus hijos.

Con respecto a la modalidad de atención en la que se desempeñan como educadoras, 65,6\% labora en Centros Infantiles del Buen Vivir y el 34,3\% en el programa "Creciendo con Nuestros Hijos". En cuanto a las características de las condiciones laborales, el $95,36 \%$ de las educadoras participantes trabaja tiempo completo, independientemente de la modalidad de atención. 71,18\% mantienen un contrato de tipo ocasional, en su gran mayoría en un horario diurno y cumpliendo la función de técnica educadora. $63,1 \%$ se mantienen en el cargo como educadora desde hace más de 2 años, pero no más de 10 atrás a la fecha del estudio, un 19,4\% labora como educadora hace 2 años o menos y el 17,3\% se desempeña como educadora desde hace más de 10 años.

Otro dato significativo en cuanto a las características socioeconómicas de las educadoras participantes fue la autopercepción del sueldo con relación a su función; el 47,8\% percibieron su sueldo como "Bajo", mientras que el 51,6\% lo considera "Mediano". El 90,6\% del total del personal en mención no han sido promovidas o ascendidas a otro cargo diferente al de educadora.

Con relación al diagnóstico luego de la aplicación del Inventario de Burnout de Maslach, se encontró que 171 participantes presentaron indicadores del síndrome de Burnout, de las cuales el $47 \%$ tuvo una leve presencia del síndrome, mientras que el $22 \%$ mostró una moderada presencia de este y un 3,38\% manifestó el síndrome de forma severa. Del total, sólo el $27,5 \%$ no presentó signos y síntomas de burnout. De las personas que presentaron el síndrome, se obtuvo como moda en la variable de cansancio emocional un puntaje de 7, lo que refiere un nivel bajo; así mismo, la generalidad de la población obtuvo un puntaje poco significativo en despersonalización y con mayor frecuencia alcanzaron un pun- taje de 42 en el área de realización personal, lo que refiere un nivel alto con tendencia a moderado.

En relación con la sintomatología del burnout, desde la lógica conversiva psicosomática, el $80,5 \%$ de las personas manifestaron somatizar el estrés a nivel de la nuca y la espalda, mientras que un 70,3\% en la cabeza; el $6,7 \%$ en las extremidades y un $5 \%$ en las partes íntimas.

Finalmente, en cuanto al conocimiento y actitud acerca de las actividades de autocuidado se obtuvo que el $95,7 \%$ del total de las educadoras encuestadas refieren conocer las actividades de autocuidado. En un porcentaje similar, el 93,22\% están de acuerdo con la realización de dichas actividades, el 95,7\% son capaces de abandonar un mal hábito y un 94,9\% pide ayuda cuando lo necesita.

En referencia a la especificidad de las actividades de autocuidado y la actitud de las educadoras con las que se realizó el sondeo, frente a dichas actividades, se obtuvo que el $78,3 \%$ practica actividad física, el 50,4\% come sanamente, el 26,6\% practica meditación. Así mismo, el 58,8\% refiere dormir lo suficiente para el descanso, un 54,6\% manifiesta acudir a un profesional de salud cuando identifica requerirlo, 53,3\% reconoce aprovechar sus pasatiempos. El 50\% del total de personas encuestadas dicen tener una vida social activa, el $49,5 \%$ refiere actividad sexual como una de las acciones de autocuidado que practica, y el $68,2 \%$ considera que dice "no" cuando lo necesita.

\section{DISCUSIÓN}

Revisando los datos obtenidos y comparándoles con resultados en otras investigaciones revisadas como la de Caballero, Bermejo, Nieto \& Caballero F. (2001), y (Arís Redó, 2009), llama la atención que en el presente estudio el $98,7 \%$ son mujeres, probablemente ello tenga que ver con la idea de que el papel del cuidado lo han asumido las mujeres, como consecuencia del cumplimiento del rol que se les ha asignado social e históricamente. Cuidar ha sido y es todavía visto como una labor de mujeres (Segarra, 2016).

El hecho de que el 61\% de las participantes, presenten un estado civil casadas o de unión libre, y que el 83,89\% del total tienen 
aún a cargo el cuidado de sus hijos representa en sí mismo la tendencia a relacionar el agotamiento evidenciado con actividades de cuidado asumidas en el hogar. Pero además con otras características sociodemográficas, como la edad promedio, la misma que osciló entre 37 años, en un rango de edad entre 20 y 58 años, lo que significaría que la mayoría de las personas participantes en el presente estudio, se hallan en una etapa de adultez media, referente al ciclo vital, en la que según Erick Erikson (2000, pp. 70-73) las personas presentan preocupación en relación con el equilibrio entre la productividad y el estancamiento. La percepción de presentar una situación económica "regular" en el caso de esta investigación que fue el $61,44 \%$ del total, son factores que por su naturaleza se relacionan con al diagnóstico de presencia del síndrome de burnout identificado.

En cuanto a la antigüedad o tiempo de trabajo revisado en las educadoras los resultados indicaron que un $63,1 \%$ se mantienen en el cargo como educadora desde hace más de 2 años, pero no más de 10 , esto significa confluyendo con el estudio sobre el síndrome de burnout en los docentes, de Nuria Arís Redó (2009) que la información levantada nos permite referirnos descriptivamente a una población con experticia, lo que a su vez asevera que las respuestas obtenidas no son consecuencia de la inseguridad que se asocia con la fase inicial en el desempeño como educador.

Con relación al diagnóstico obtenido a través de la aplicación del MBI a las educadoras participantes de este estudio es relevante que un $47 \%$ y un $22 \%$ muestre una leve y moderada presencia de este respectivamente, estos resultados se contrastan con algunas investigaciones en los que se ha presenciado con mayor frecuencia el nivel moderado y alto del síndrome, como en Caballero et al. (2001), o en Solís, Tantalean, Burgos \& Chambis (2017). Sin embargo, existe confluencia con otros estudios en los que los puntajes obtenidos en las variables que explora el MBI, como en (Cebrià, y otros, 2001) que obtuvieron en su estudio que en Realización Profesional (RP) se destacó la media (66\%) seguida de baja (21\%) Realización Profesional. En la presente investigación, de las personas que presentaron el síndrome, se obtuvo como moda en la variable de cansancio emocional un puntaje de 7 , lo que refiere un nivel bajo, así mismo, la generalidad de la población obtuvo un puntaje poco significa- tivo en despersonalización y con mayor frecuencia alcanzaron un puntaje de 42 en el área de realización personal, lo que refiere un nivel alto con tendencia a moderado.

Otro importante dato en la presente investigación radica en que el 90,6\% del personal participante, indicó que no han sido promovidas o ascendidas a otro cargo diferente al de educadora, durante el tiempo laborado hasta el momento, lo que podría indicar que este muy probablemente sea un factor asociado al cansancio emocional debido a la instalación de la rutina. Otro dato relevante y que coincide con las características laborales de la muestra en la investigación de Almeida, y otros (2015) es que en su mayoría las educadoras en mención laboran bajo la modalidad de un contrato ocasional, lo que podría relacionarse con la tensión psicológica que se genera ante la incertidumbre de las formalidades que garantizan la estabilidad laboral; así mismo con la consideración de un sueldo percibido como "mediano" y "bajo" en relación al quehacer en el trabajo, factores que probablemente coincida con la presencia leve del síndrome de burnout.

Un porcentaje de importancia es además que el $80,5 \%$ de las personas manifiestan somatizar el estrés a nivel de la nuca y la espalda, mientras que un $70,3 \%$ en la cabeza, esto indica que estas son las zonas de cuerpo que mayormente se ven comprometidas ante el desarrollo de posibles enfermedades psicosomáticas relacionadas con la severa presencia del síndrome de burnout.

Así mismo, la cifra obtenida nos ha permitido comprender que, aunque el 95,7\% son capaces de abandonar un mal hábito y un 94,9\% pide ayuda cuando lo necesita, las actividades de autocuidado no se llevan a la práctica en un porcentaje significativo, lo que llevaría a pensar en el estado de conciencia acerca de la necesidad de incorporar variedad y frecuencia en cuanto a la práctica de actividades de autocuidado.

\section{CONCLUSIONES}

La presente investigación ha permitido ampliar la comprensión del Síndrome de Burnout en las Educadoras Familiares de Manabí, constituyendo así los primeros bosquejos para ahondar en la contribución de la salud mental y la calidad de vida de las personas participan- 
tes en el presente estudio y al buen vivir en relación con los entornos intervinientes en la cotidianidad de las educadoras.

Esta investigación posibilita una reflexión acerca de la tendencia a presentar el síndrome de Burnout en las educadoras de CIBV y $\mathrm{CNH}$ del Ministerio de Inclusión Económica y Social del Ecuador. Y sugerir próximas investigaciones acerca de la relación entre los hallazgos en mención y las variables socioeconómicas descritas. A través del análisis cuanti-cualitativo se pudo observar que la mayoría de las educadoras participantes del presente estudio presentó un diagnóstico de leve presencia del síndrome de burnout, caracterizados por un puntaje de 7 , lo que refiere un nivel bajo de cansancio emocional, así mismo, la generalidad de la población obtuvo un puntaje poco significativo en despersonalización y con mayor frecuencia alcanzaron un puntaje de 42 en el área de realización personal, lo que refiere un nivel alto con tendencia a moderado.

Esto lleva a la conclusión que las cifras obtenidas perfilan un tamiz de riesgo a desarrollar la presencia del síndrome en niveles considerados moderados. Además, ya que hay un porcentaje del $8 \%$ que presenta de forma severa el síndrome. Aunque algunas educadoras realizan actividades de autocuidado, ello no representa un porcentaje significativo según el presente estudio, se sugiere que en posteriores investigaciones se profundice no solo en la frecuencia y variedad de actividades de autocuidado, sino también en cuanto a la calidad de la realización de dichas actividades, es decir las condiciones en las que desempeñan dichas acciones.

Se espera con esta investigación facilitar a las instituciones pertinentes, una perspectiva de la salud mental de las educadoras de las modalidades de atención a niñas y niños, que por un lado permita comprender e intervenir en beneficio de la promoción de la salud, y la creación de las políticas públicas respectivas.

Se considera que, ante los hallazgos de otras investigaciones relacionadas con la presencia del síndrome de burnout en educadoras en las modalidades de atención competentes del MIES en el país, se investigue la relación posible entre los resultados diagnósticos del síndrome y las variables socioeconómicas y demográficas tomando en cuenta la diversidad cultural del Ecuador.

Finalmente, se espera que el presente estudio no solo promueva posteriores debates académicos en torno al tema en mención, sino también que pueda servir para la detección precoz del síndrome y el planteamiento de incursionar en la estructuración de propuestas relacionadas al abordaje de la realidad referida. 


\section{REFERENCIAS BIBLIOGRÁFICAS}

Almeida, M., Nazaré, E., Guimarães, F., Evangelista, R., Gomes, A., \& Vieira, B. (2015). Síndrome de Burnout: un estudio con profesores. Salud de los Trabajadores, 23(1), 19-27.

Arís Redó, N. (2009). El Síndrome de Burnout en los docentes. (U. d. Almería, Ed.) Electronic Journal of Research in Educational Psychology, 7(18), 829-848. Obtenido de http://www.redalyc.org/articulo. oa?id=293121945012

Barragán, G. (25 de febrero de 2016). Realidad laboral: Ecuador no tiene políticas de prevención contra el burnout. No existen estadísticas reales. Redacción Médica. (C. Coello, Entrevistador) Quito: Sanitaria 2000. Recuperado el 16 de Enero de 2018, de https://www. redaccionmedica.ec/secciones/salud-publica/ecuadorno-tiene-pol-tica-de-prevenci-n-contra-elburnout-87258

Brito, J., Cabrera, H., \& Cedilllo, J. (2012). Prevalencia y factores influyentes del síndrome del Burnout en internos de los hospitales Vicente Corral y José Carrasco (tesis de grado en medicina). Cuenca, Azuay, Ecuador: Universidad de Cuenca.

Caballero, M., Bermejo, F., Nieto, G., \& Caballero, F. (2001). Prevalencia y factores asociados al burnout en un área de salud. (C. d. Escorial, Ed.) Atención Primaria, 313317. Obtenido de http://www.bvsde.paho.org/bvsacd/ cd49/burnout.pdf

Caicedo A., C. E. (2018). Identificación del burnout en profesionales de la salud y factores relacionados con este fenómeno en el Ecuador 2017. (Trabajo de grado). Quito: UTPL.

Camacho E, V.-M. C. (2014). Autocuidado de la salud. (1 ${ }^{\text {a }}$ ed.). Guadalajara: Instituto Tecnológico y de Estudios Superiores de Occidente (ITESO).

Cárdenas, J. (2013). Prevalencia de S. de burnout y su influencia en la Realización personal en los profesionales de las áreas críticas del hospital del instituto ecuatoriano de seguridad social de la ciudad de Ambato (Tesis de grado en medicina). Ambato, Tungurahua, Ecuador: Universidad de Ambato.

Cebrià, J., Segura, J., Corbella, S., Sos, P., O., C., Rodríguez, C., .. . García-Simal, M. (2001). Rasgos de personalidad y burnout en médicos de familia. Atención Primaria, 27(7), 459-468. Obtenido de https://doi. org/10.1016/S0212

Clipa, O. (2017). Studies and Current Trends in Science of Education. Teacher Stress and Coping Strategies. Lumen proccedings. Suceava, 120-128. doi:DOI: 10.15611/eada.2018.1.02

Cooper, S., Carleton, H., Chamberlain, S., Cummings, G., Bambrick, W., \& Estabrooks, C. (2016). Burnout in the nursing home health care aide: A systematic review . Elsevier, 3(3), 76-87.

Costales, L. (2018). La resiliencia relacionada con el desarrollo del síndrome de burnout en residentes del posgrado de pediatría de la PUCE (tesis de postgrado). Quito, Ecuador: Pontificia Universidad Católica del Ecuador.

Cruz, C., Guanga, K., \& León, J. (2014). Influencia del estrés (síndrome de burnout) en el desempeño laboral de los profesionales médicos del hospital del seguro social (IESS) del cantón Machala año 2014 (tesis de pregrado). Machala, El Oro, Ecuador: UTMACH, Unidad Académica de Ciencias Sociales.

Díaz B., F., \& Gómez, I. C. (2016). La investigación sobre el síndrome de burnout en latinoamérica entre 2000 y el 2010. Psicología desde el caribe, 33(1), 113-131. Recuperado el 2016, de http://dx.doi.org/10.14482/ psdc.33.1.8065

Díaz, P., Radler, E., Amadeo, M., \& Guerrero, K. (2011). "El burnout" ;está entre nosotros? Hospital Municipal de Avellaneda "Dr. Eduardo Wilde", Ginecología. Avellaneda: S/N. Recuperado el 5 de Diciembre de 2016, de http:// sedici.unlp.edu.ar/bitstream/handle/10915/43666/
Documento_completo.pdf?sequence $=3$

Erikson, E. (2000). El ciclo vital completado. (R. Maluquer, Trad.) Barcelona, España : Paidós Ibérica.

Freudenberger, H. (1974). Staff burnout. Journal of Social Issues, 30(1), 159-165. Recuperado el 15 de 10 de 2017, de http://onlinelibrary.wiley.com/ doi/10.1111/j.1540-4560.1974.tb00706.x/abstract

García, M., Delgado, E., \& Lazo, M. (2018). Síndrome de Burnout y la atención al público en el hospital Verdi Cevallos Balda, Portoviejo". Revista Caribeña de Ciencias Sociales, En línea. doi:https://www.eumed.net/rev/ caribe/2018/09/sindrome-burnout-hospital.html

Garzón, L. (2017). Análisis correlacional entre las fuentes y los mecanismos de afrontamiento de estrés laboral en la empresa Electrobahía Cia. Ltda. de la ciudad de Riobamba del Ecuador. Quito, Pichincha, Ecuador: Escuela Politecnica Nacional.

Gómez, A., Algora, A., Suasnavas, P., Silva, M., \& Vilaret, A. (2016). Notificación de Accidentes de Trabajo y Posibles Enfermedades Profesionales en Ecuador, 20102015. Ciencia \& trabajo, 18(57), 166-172. Obtenido de https://dx.doi.org/10.4067/S0718-24492016000300166

Hall, L., Johnson, J., Watt, I., Tsipa, A., \& O'Connor, D. (2016). Healthcare Staff Wellbeing, Burnout, and Patient Safety: A Systematic Review. (F. Harris, Ed.) Plos One, USA, 11(7), 1-12. doi:DOI:10.1371/journal.pone.0159015

Hernandez Sampieri, R., Fernández, C., \& Baptista, P. (2010). Metodología de la investigación (5ta. ed.). México: McGraw Hill.

López-Pozos, C. (Julio-Diciembre de 2014). Síndrome de Burnout estudiantil y género. Ra Ximhai, 10(7), 185-202. Recuperado el 28 de Noviembre de 2016, de http://www. redalyc.org/articulo.oa? $\mathrm{id}=46132451013$

Maslach, C., \& Jackson, S. (Abril de 1981). The measurement of experienced burnout. . Journal of Occupational Behaviour, 2, 99-113. doi:10.1002/ job.4030020205

Menéndez, T., Calle, A., Delgado, D., Espinel, J., \& Cedeño, Y. (2018). Síndrome de Burnout y calidad de atención del personal de enfermería de un hospital general en la provincia de Manabi, Ecuador. Contribuciones a las Ciencias Sociales, En línea:. Obtenido de https://www.eumed.net/rev/cccss/2018/10/ sindrome-burnout-ecuador.html

Ministerio de Inclusión Económica y Social. (2015). Informe de Gestión 2015. Manabí: MIES. Obtenido de http://www.inclusion.gob.ec/documentos-rendicion-decuentas-2015/

Ministerio de Inclusión Económica y Social. (01 de Enero de 2015). Ministerio de Inclusión Económica y Social Recuperado el miércoles 04 de Enero de 2017, de Programa de Capacitación Continua: http://186.46.86.234/formacioncontinuadi/

Moreno-Jiménez, B., González, J. L., \& Garrosa, E. (2001). Desgaste profesional (burnout), Personalidad y Salud percibida . (D. P. UAM, Ed.) Empleo, estrés y salud. , 5983. Recuperado el 4 de Enero de 2017, de https://www. uam.es/gruposinv/esalud/Articulos/Personalidad/

Desgaste\%20profesional-personalidad-y-saludpercibida.pdf

Pando, M., Aranda, C., \& López, M. (2015). Validez factorial del Maslach Burnout Inventory-General Survey en ocho países Latinoamericanos. Ciencia \& Trabajo, 17(52), 28-31. Obtenido de http://dx.doi.org/10.4067/ S0718-24492015000100006

Pérez, R. (2017). Impacto del Burnout sobre el desempeño laboral en operarios de una empresa petrolera de Ecuador (tesis de grado en psicología). Quito: Universidad de las Américas, UDLA.

Pesántez, M. (2015). Prevalencia del síndrome de burnout y propuesta de una estrategia para su control en los médicos del distrito de salud 01D06, Ecuador, 2015. 
REFERENCIAS BIBLIOGRÁFICA (CONT.)S

Cuenca, Ecuador: Universidad del Azuay. Obtenido de http://dspace.uazuay.edu.ec/bitstream/ datos/5899/1/12219.pdf

Prado, A., González, M., Paz, N., \& Romero, K. (2014). LaTeoría Déficit de autocuidado: Dorothea Orem punto de partida para calidad en la atención. Revista Médica Electrónica, 36(6), 835-845.

Quevedo, B. (2012). Identificación del burnout en profesionales de la salud y factores relacionados con este fenómeno, en el Hospital República del Ecuador, Unidad Ambulatoria del Seguro Social y médicos Particulares, Santa Cruz, Galápagos, 2011.2012. Santa Cruz, Ecuador: UTPL.

Quiceno, J., \& Vinaccia, S. (julio-diciembre de 2007) Burnout: síndrome de quemarse en el trabajo (SQT). Acta Colombiana de Psicología(100), 117-125.

Ramírez, M. (2015). Burnout en profesionales de la salud del Ecuador (tesis doctoral). Santiago de Compostela: Universidad Santiago de Compostela.

Ramírez, M. (2017 a). Síndrome de Burnout en Profesionales de la Salud en a Zona 7 de Ecuador. Perspectivas UTPL, 8-9.

Ramírez, M. (2017 b). Prevalencia del síndrome de burnout y la asociación con variables sociodemográficas y laborales en una provincia de Ecuador. International Journal of Developmental and Educational Psychology, 4(1), 241-251. doi:http://www.redalyc.org/articulo. oa?id=349853537025

Saborío, L., \& Hidalgo, L. (Marzo de 2015). Síndrome de Burnout. Medicina Legal de Costa Rica, 32(1), 119-124.

Segarra F., O. (2016). El Estrés laboral en educadoras de los Centros Infantiles del Buen Vivir urbanos y Rurales de la ciudad de Cuenca 2013 - 2014. Cuenca: Universidad del Azuay.

Solís, R., Tantalean, M., Burgos, R., \& Chambis, J. (2017). Agotamiento profesional: prevalencia $y$ factores asociados en médicos y enfermeras en siete regiones del Perú. , ),. Anales de la Facultad de Medicina, 78(3), 270276. https://dx.doi.org/10.15381/anales.v78i3.13757

Vidal, F., Nicasio, J., \& Pacheco, D. (2010). El burnout en los profesores. International Journal of Developmenta and Educational Psychology, 1(1), 251-256. Obtenido de https://www.redalyc.org/articulo.oa? $i d=349832324027$

Vilcahuano Germán, D. (2015). El clima organizacional y su relación con el síndrome de burnout, en los servidores de la Dirección de Talento Humano de la Universidad Central del Ecuador (tesis de grado). Quito: Universiidad Central del Ecuador.

Vivanco, M., Sánchez, C., Maldonado, R., \& Erique, E. (2018). Síndrome de burnout en profesionales de la salud en instituciones públicas y privadas: un análisis en la provincia de Loja-Ecuador. Redipe, 7(11), 179-194. Obtenido de https://revista.redipe.org/index.php/1/ article/view/622

Yawen, S., \& Zhen, F. (2018). The Influence of Self-worth on the Burnout of Health Care Teachers in Primary and Secondary Schools in Shanghai., . DO. London International Conference on Education and Cognition, Behavior, Neuroscience (págs. 149-152). Londres: Francis Academic Press. doi:DOI: 10.25236/icecbn.2018.023

Zaluska, U., Ślazyk-Sobol, M., \& Kwiatkowska-Ciotucha, D. (2018). Burnout and its correlates-an empirical study conducted among education, higher education. Ekonometria. Econometrics, 22, 26-38. 